\title{
portance réelle et portance calculée des pieux isolés, sollicités verticalement
}

\author{
Règles pressiométriques
}

\author{
par \\ X. Bustamante \\ Dr. Ingénieur \\ L. Gianeselli \\ Section des Fondations \\ Département des Sols et Fondations \\ Laboratoire Central des Ponts et Chaussées-Paris.
}

\section{Introduction}

Déterminer la capacité portante d'un pieu de manière à ce que celle-ci soit très proche de sa portance réelle, reste toujours très difficile. Cela s'explique par le fait que la presque totalité des méthodes de calcul actuelles ont été élaborées sans tenir compte d'un élément aussi essentiel que l'incidence de la mise en œuvre sur la portance mais surtout, parce que les valeurs des différents paramètres de calcul que font intervenir ces méthodes, n'ont été étalonnées qu'à partir d'un nombre trop faible de pieux réels! De plus, le fait que ces derniers n'aient même pas été instrumentés ou, lorsqu'ils l'étaient, médiocrement, a conduit beaucoup de praticiens à tirer des conclusions erronées sur le comportement des pieux et, par voies de conséquence, à proposer des valeurs de paramètres de calculs souvents assez éloignées de la réalité.

Une étude de synthèse, reposant sur l'interprétation d'un total de 192 essais $^{*}$ de chargements en vraie grandeur a conduit, après comparaison des portances réelles et calculées selon la méthode pressiométrique, à proposer un réajustement de cette dernière. On verra aussi par la suite, que celle-ci a fait l'objet de quelques développements.

\begin{tabular}{l|c|c|c}
\hline $\begin{array}{c}\text { Types de } \\
\text { fondations } \\
\text { profondes }\end{array}$ & $\begin{array}{c}\text { Essayées par } \\
\text { les L.P. C. }\end{array}$ & $\begin{array}{c}\text { Essayées par } \\
\text { différents } \\
\text { organismes }\end{array}$ & Total \\
\hline Forées & 39 & 15 & 54 \\
Battues & 17 & 14 & 31 \\
Injectées & 8 & 0 & 8 \\
Barrettes & 0 & 1 & 1 \\
Puits & 1 & 0 & 1 \\
\hline
\end{tabular}

Tableau 1

*On gardera à l'esprit qu'un même pieu a pu faire l'objet de plusieurs chargements.
En ce qui concerne les essais, il faut rappeler que si ceux-ci ont été effectués en majeure partie par le réseau des Laboratoires des Ponts et Chaussées, on a également utilisé les résultats de chargements réalisés par différents organismes (bureaux de contrôle, entreprises, instituts de recherches) tant en France qu'à l'étranger [1] [2] [3] [4].

Les essais ont intéressé un total de 45 sites* regroupant des sols aussi divers que les argiles, limons, sables, graves ou même rochers altérés mais également des vases, des tourbes, des craies plus ou moins altérées, des marnes. Le tableau 1 donne un aperçu des types de fondations pris en compte. On constate que les pieux forés y sont le mieux représentés (plus de $70 \%$ du total) ce qui s'explique par la prédominance actuelle de ce type de fondations en France. Point important, la quasi-totalité de ces fondations a été mise en œuvre par des entreprises de fondations spécialisées suivant les techniques courantes de chantier.

II faut faire remarquer, que les pieux forés (tarière, bucket, hammergrab, trépan, soupape) regroupaient des pieux forés simples, tubés en totalité ou en partie, forés à la boue. Les pieux battus (ou vérinés) regroupaient des pieux métalliques $\mathrm{H}$ ou tubulaires à base fermée, en béton armé (section pleine) et dans un cas même, une palplanche.

Le tableau 1 montre toutefois que les barrettes ou certains types de pieux figurant dans le dernier DTU «Fondations profondes " [5] ne sont pratiquement pas représentés ou sont carrément absents. II s'agit pour ces derniers:

- des pieux tubulaires précontraints ou tubulaires métallique à base ouverte,

- des pieux battus moulés,

- des pieux vissés moulés,

- des micropieux de type I ou II.

*à raison de 36 pour les Laboratoires des $P$. et $C h$. et 9 pour les divers organismes. 
Néanmoins, au niveau des propositions ultérieures, on se prononcera sur le mode de calcul de ces quelques pieux, considérant que leur mode de travail s'apparente suffisamment à celui de fondations dont on a pu étudier expérimentalement le comportement.

\section{Les essais de sol}

Sur les 36 sites contrôlés par les Laboratoires des Ponts et Chaussées, on s'est efforcé, préalablement à la mise en place des pieux ou leur chargement, de réaliser la gamme complète d'essais de reconnaissance des sols : pressiométriques (sonde Ménard) et pénétrométriques statiques (ou dynamiques) mais aussi de laboratoire lorsque le prélèvement d'échantillons intacts était possible. Le tableau 2 donne pour l'ensemble de ces sites, une bonne idée de la faisabilité et de la représentative des différents types d'essais.

L'examen du tableau 2 est extrêmement parlant, il montre bien tout l'intérêt de l'essai pressiométrique standard (Ménard). Quel que soit la nature du sol rencontré, de l'importance des couches et de leur alternance, l'essai pressiométrique s'avère être dans la pratique courante*, toujours réalisable, exploitable et suffisamment représentatif.

Quand aux essais pénétrométriques ou de laboratoire, en dépit de l'intérêt qu'ils peuvent présenter, la nature d'une partie importante des sols rencontrés sur le territoire français ${ }^{\star \star}$ du fait de leur structure complexe (blocs, cimentations ou concrétions), des taux d'altération élevés (craies, roches), du manque de cohésion (sols pulvérulents propres) ou tout simplement des compacités importantes, en limite fortement la mise en œuvre.

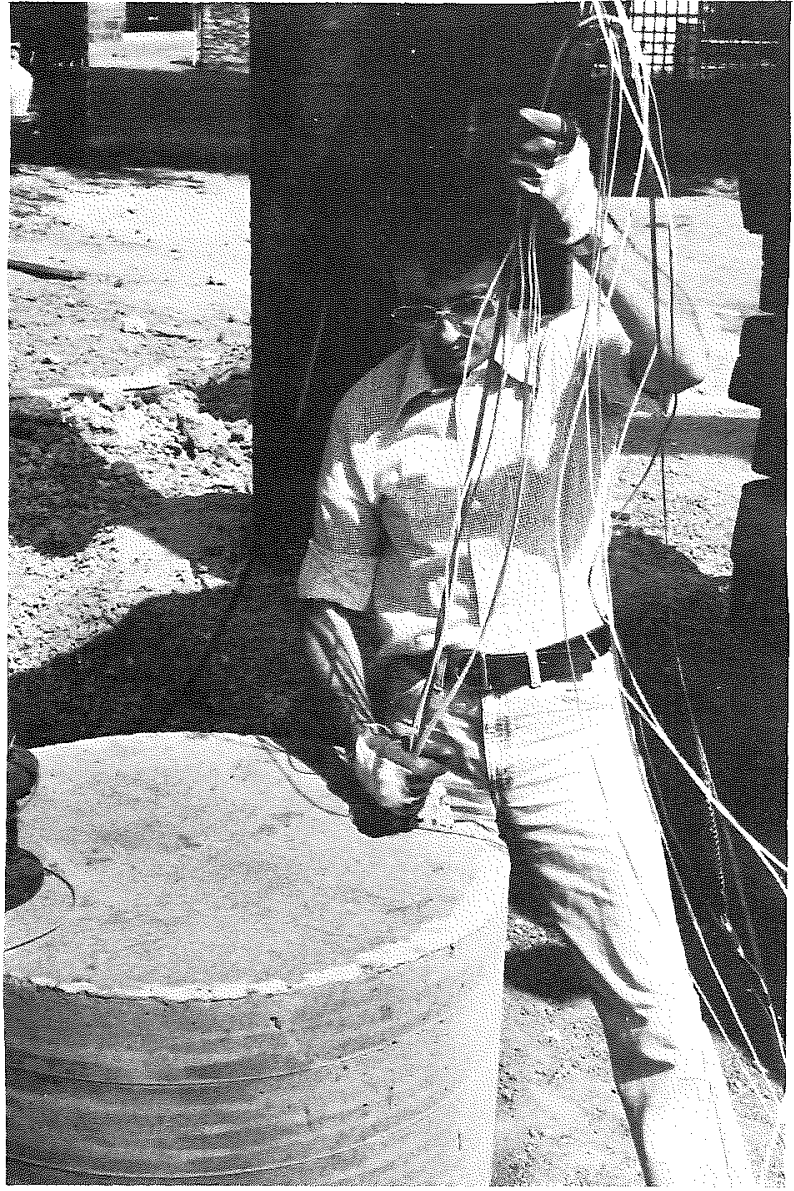

Fig. 1 Opération de mise en place d'un extensomètre amovible à bloqueurs (modèle 72) dans un pieu foré simple. Site de Roubaix

\begin{tabular}{|c|c|c|c|c|c|c|}
\hline $\begin{array}{l}\text { Types d'essai } \\
\text { entrepris (para- } \\
\text { mètre caractéris- } \\
\text { tique) }\end{array}$ & $\begin{array}{l}\text { Total } \\
\text { des } \\
\text { sites }\end{array}$ & $\begin{array}{l}\text { Essais effecti- } \\
\text { vement réalisés }\end{array}$ & $\begin{array}{l}\text { Essais jugés ex- } \\
\text { ploitables ou } \\
\text { représentatifs } \\
\text { après coup }\end{array}$ & $\begin{array}{c}\text { Essais considérés } \\
\text { partiels ou inex- } \\
\text { ploitables (1) }\end{array}$ & $\begin{array}{c}\text { Essais jugés } \\
\text { non réalisables } \\
\text { a priori }\end{array}$ & $\begin{array}{l}\text { Essais possi- } \\
\text { bles et of- } \\
\text { frant la cer- } \\
\text { titude de la } \\
\text { représentati- } \\
\text { vité }\end{array}$ \\
\hline $\begin{array}{c}\text { pénétromètre } \\
\text { statique } \\
\left(q_{c}\right)\end{array}$ & 36 & 18 & 10 & $\begin{array}{c}8 \\
\text { (refus prématuré) }\end{array}$ & 16 & 2 \\
\hline $\begin{array}{l}\text { pressiomètre } \\
\text { Ménard } \\
(p . l)\end{array}$ & 36 & 34 & 31 & $\begin{array}{c}3 \\
\text { (mesures trop es- } \\
\text { pacées) }\end{array}$ & 0 & 2 \\
\hline $\begin{array}{l}\text { laboratoire } \\
\qquad(c, \varphi)\end{array}$ & 36 & 16 & 7 & $\begin{array}{c}9 \\
\text { (trop grande } \\
\text { dispersion) }\end{array}$ & 14 & 6 \\
\hline
\end{tabular}

(1) La raison principale figurant entre parenthèses.

\section{Tableau 2}

*sites terrestres évidemment et profondeurs n'excédant pas les 40 mètres.
*considération que les auteurs n'hésiteraient pas à extrapoler à la presque totalité des sols étrangers. 


\section{La méthodologie des essais de chargement}

L'ensemble des résultats qui ont été utilisés pour établir les nouvelles règles pressiométriques provenait de l'interprétation d'essais de chargements verticaux statiques. Les pieux essayés étant bien évidemment axialement sollicités.

Dans le cas où un même pieu avait fait l'objet de plusieurs essais, il n'a été tenu compte que des résultats provenant du premier chargement. Cela pour éliminer l'incidence du facteur temps [6].

Au total, les fûts de 53 pieux (28 sites) ont été instrumentés dans le but de pouvoir établir entre autres, la part d'effort reprise par la résistance de pointe et le frottement latéral. Équipées quelquefois de jauges collées ou de témoins sonores, très rarement de cellules de contraintes ${ }^{*}$, dans la majorité des cas et en raison des avantages indiscutables qu'ils présentent, les fondations ont été instrumentées à l'aide d'extensomètres amovibles à bloqueurs ou à tripodes [7] [8]. La figure 1 offre une vue de l'un de ces appareils en cours d'installation dans un pieu foré simple. Chaque fois que cela a été possible, vu l'incidence de la valeur du module d'élasticité $E$ du matériau constitutif de la fondation sur l'évaluation des efforts, notamment pour les pieux forés, on a mesuré ce paramètre sur des éprouvettes prélevées directement au sein du fût, après confection de ce dernier. La figure 2 donne un exemple de la variation du module d'élasticité E le long de deux fûts de pieu foré à la boue et d'un pieu battu préfabriqué.

Point important, la totalité des essais de chargement pris en compte et réalisés en France, a été conduite suivant les directives du Mode Opératoire de l'Essai Statique de L.P.C. [9], repris par le dernior D.T.U. "Fondations Profondes» [5]. On rappellera que selon ces 2 documents, l'essai consiste à éprouver une fondation par paliers de charges croissants d'égales intensités et durées ( 60 ou 90 minutes), sans déchargements intermédiaires. L'importance de chacun des paliers est de l'ordre du $1 / 10^{e}$ de la charge limite calculée $Q_{L}$ (fig. 3 a). Au niveau de l'interprétation, il est prescrit de tracer la relation caractéristique $\alpha-Q$ (fig. 3 b) de laquelle on déduit la charge critique de fluage $Q_{F}$, délimitant en principe deux régimes de fluage du pieu. Un premier domaine pour lequel les fluages ou, ce qui revient au même, les déplacements y du pieu sous charge constante, restent extrêment faibles et linéaires ${ }^{\star \star}$, un second domaine pour lequel l'amplitude et la vitesse des déplacements y deviennent importantes et sont susceptibles d'augmenter à plus ou moins long terme [1] [10] [11].

\section{Rappel des principes de la méthode pressio- métrique}

II convient avant de proposer toutes nouvelles règles, de rappeler brièvement les quelques principes sur lesquels repose la méthode pressiométrique énoncés par Ménard [12] [13] et repris pratiquement dans leur intégralité par le document FOND. 72 [17].

La charge limite calculée $Q_{L}$ d'une fondation profonde est la somme de 2 termes (fig. 4) :

$$
Q_{L}=Q_{L}^{P}+Q_{L}^{F} \quad[k N]
$$

\footnotetext{
*Mesure directe des efforts.

**Donc extrapolables et prévisibles.
}

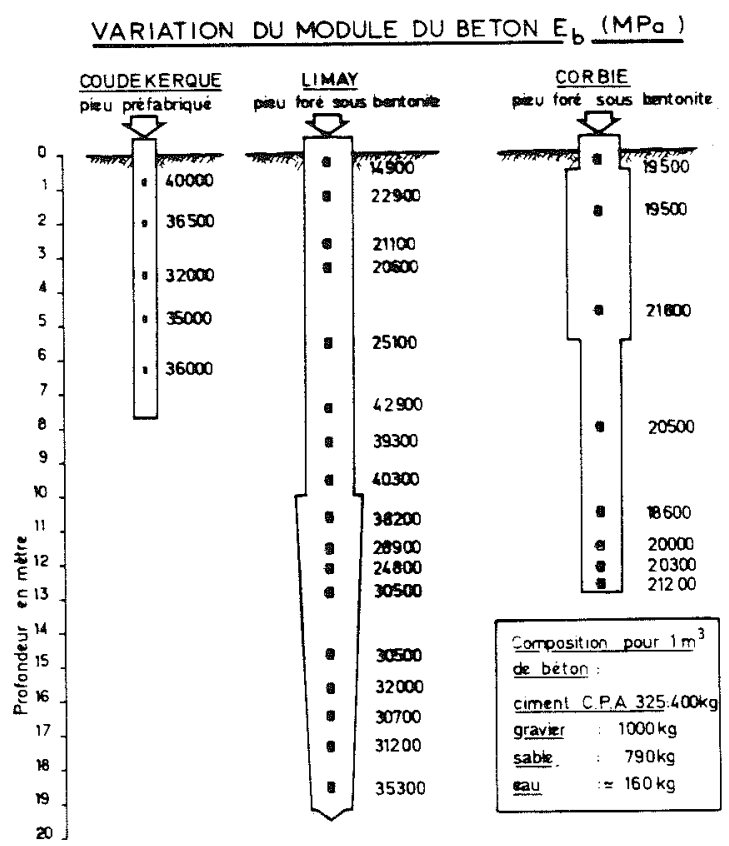

Fig. 2

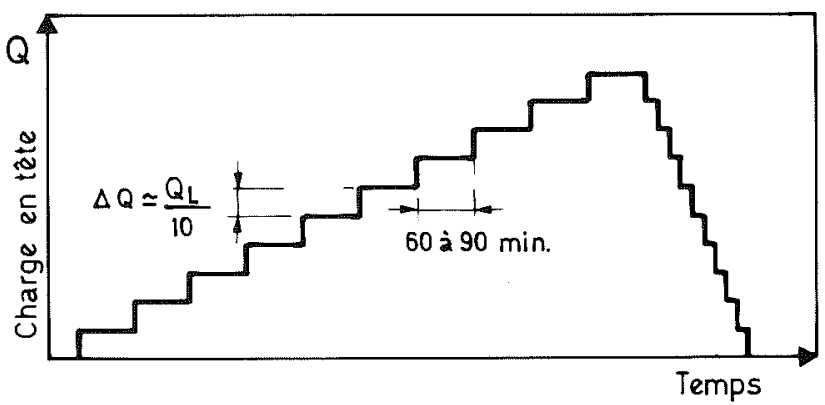

Fig. 3a Schématisation du programme de chargement seIon le mode opératoire des $L P C$

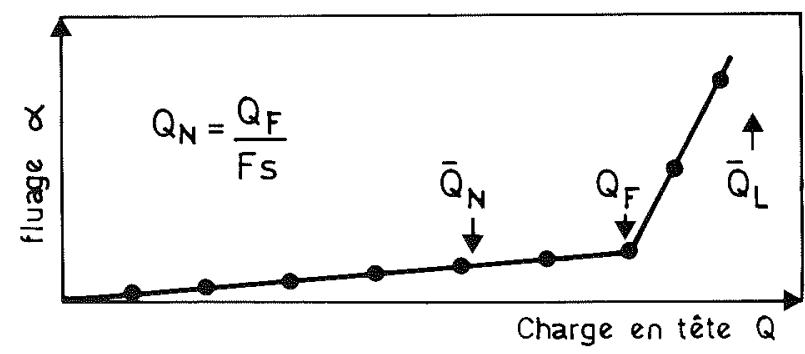

Fig. 3b Rappel de la construction graphique pour la détermination de la charge critique de fluage $Q_{F}$

où :

$Q_{L}^{P} \quad$ - la résistance limite sous la pointe $[\mathrm{kN}]$

$Q_{L}^{F} \quad$ - le frottement limite sur toute la hauteur du fût de la fondation [kN].

Dans le cas général d'un multicouche pour lequel on connaît en fonction de la profondeur la distribution des pressions limites $p_{\text {, }}$ (fig. 4), chacun de ces termes sera calculé à partir des formules ci-après :

$$
\begin{aligned}
& Q_{L}^{P}=\left[q_{0}+k\left(p_{\ell e}-p_{0}\right)\right] \cdot \frac{\pi D^{2}}{4}[k \pi] \\
& Q_{L}^{F}=\sum_{p}^{i} Q_{L i}^{F}=\sum_{\ell}^{i} q_{s i} \pi D \ell_{i} \quad[k N]
\end{aligned}
$$



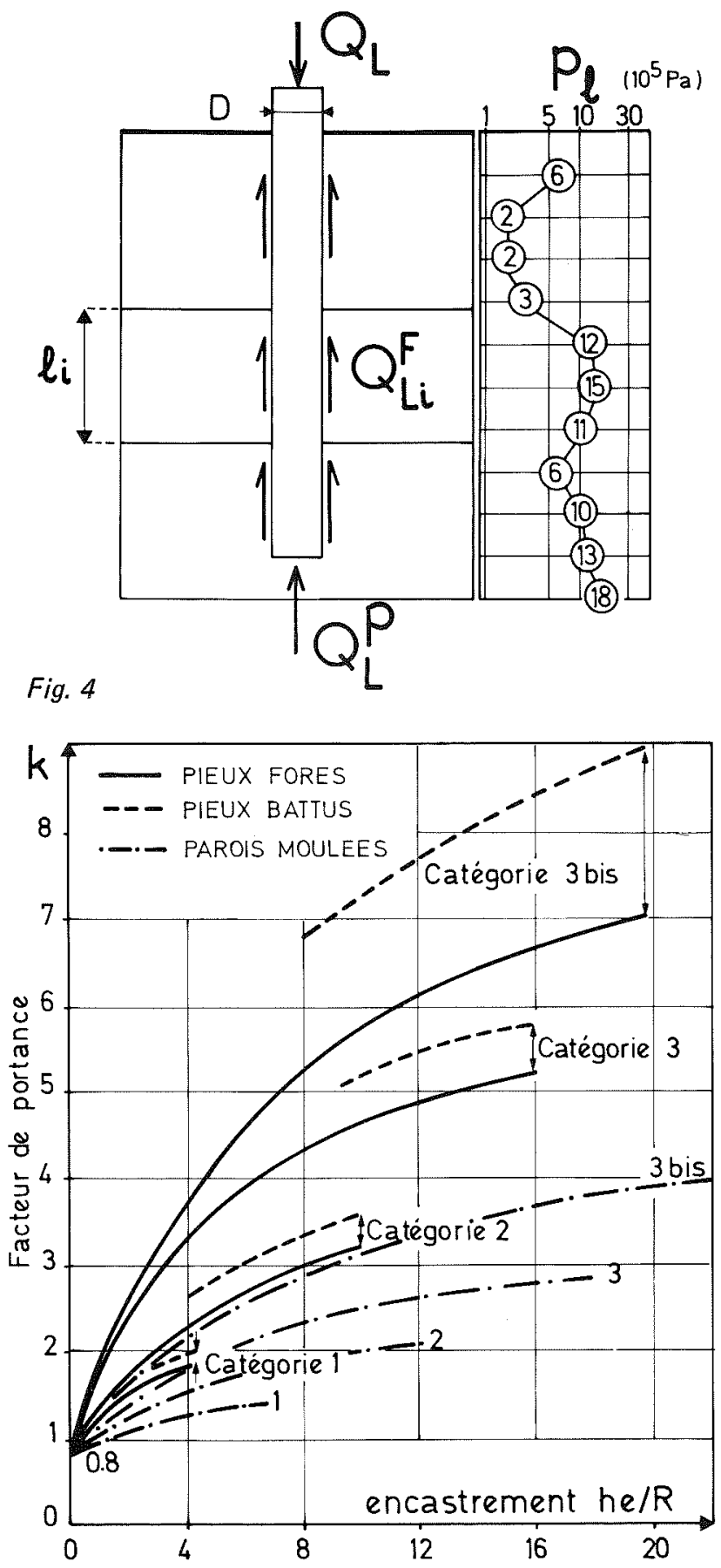

où successivement :

$q_{o}$ - la pression verticale totale des terres au niveau de la pointe $\left[\mathrm{kN} / \mathrm{m}^{2}\right]$

k - le facteur de portance pressiométrique

$\mathrm{p}_{\ell e}$ - la pression limite équivalente au niveau de la pointe $\left[\mathrm{kN} / \mathrm{m}^{2}\right.$ ]

Po - la pression horizontale totale des terres au niveau de la pointe $\left[\mathrm{kN} / \mathrm{m}^{2}\right]$

[. - le diamètre de la fondation [m]

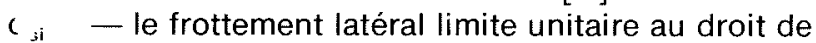
la couche $i$

$t_{1} \quad$ - l'épaisseur de la couche $i[\mathrm{~m}]$.

S'il paraît superflu de commenter la plupart des paramètres entrant dans les formules, il y a lieu d'insister sur la manière de déterminer le facteur $k$ et le frottement unitaire $\mathrm{q}_{\mathrm{s}}$. Ils font à eux deux presque toute l'originalité de la méthode. Ils en conditionnent aussi toute la validité. Comme on pourra le constater par la suite, le réajustement proposé visera essentiellement ces 2 paramètres.

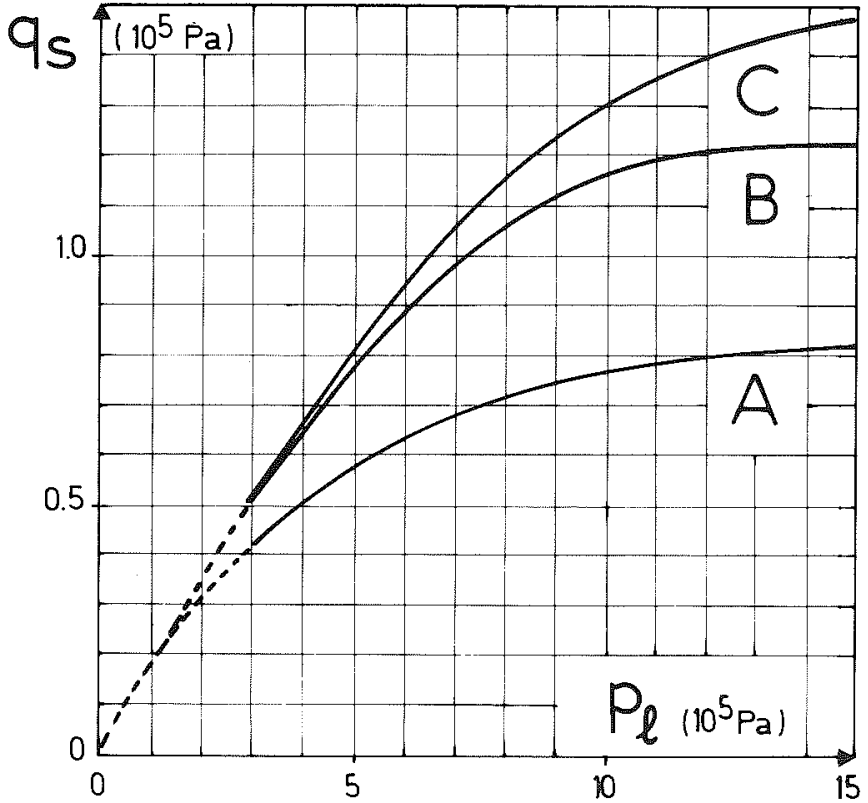

Fig. 6 Anciennes règles pressiométriques. Abaques pour la détermination du frottement unitaire $q_{s}$

\begin{tabular}{|c|c|c|}
\hline $\begin{array}{l}\text { Plages des pressions } \\
\text { limites } \mathrm{pl}\end{array}$ & Nature du sol & Catégories de sol \\
\hline $\begin{array}{l}0-12 \text { bar } \\
0-7\end{array}$ & $\begin{array}{l}\text { Argile } \\
\text { Limon }\end{array}$ & catégorie I \\
\hline $\begin{array}{c}18-40 \\
12-30 \\
4-8 \\
10-30\end{array}$ & $\begin{array}{l}\text { Argile raide et marne } \\
\text { Limons compacts } \\
\text { Sable compressible } \\
\text { Roche tendre ou altérée }\end{array}$ & catégorie II \\
\hline $\begin{array}{l}10-20 \\
40-100\end{array}$ & $\begin{array}{l}\text { Sable et gravier } \\
\text { Roche }\end{array}$ & catégorie III \\
\hline $30-60$ & $\begin{array}{l}\text { Sable et gravier } \\
\text { très compact }\end{array}$ & catégorie III bis \\
\hline
\end{tabular}

Fig. 5 Anciennes règles pressiométriques

a) abaques pour la détermination du facteur de portance $k$ b) tableau des catégories de sol

Le facteur de portance $k$, qui est déterminé à partir d'abaques (fig. 5 a) est fonction de la nature du sol et de sa compacité (notion de catégorie, fig. $5 \mathrm{~b}$ ), de l'encastrement relatif de la fondation (paramètre $h_{e}$ ), enfin du mode de mise en œuvre du pieu (battu, foré).

L'estimation $\mathrm{du}$ frottement unitaire $\mathrm{q}_{\mathrm{s}}$ s'effectue également à partir d'abaques (fig. 6) tenant compte de la nature du fût de la fondation (acier et béton), de son mode de mise en œuvre (forage ou battage), enfin de la nature et de la compacité $\left(\mathrm{p}_{\ell}\right)$ du sol. Dans certains cas, il est prescrit d'appliquer un abattement (25 à $50 \%$ ) aux valeurs données par les abaques.

Enfin, la valeur de la charge nominale calculée $Q_{N}$ du pieu s'obtient en adoptant un coefficient de sécurité de 3 pour la résistance de pointe et de 2 pour le frottement latéral soit :

$$
Q_{N}=\frac{Q_{L}^{P}}{3}+\frac{Q_{L}^{F}}{2} \quad[k N]
$$




\section{Conventions retenues au niveau de l'inter- prétation des essais de chargement}

On a défini la charge limite réelle $\bar{Q}_{L}$ de la fondation comme étant la charge d'essai correspondant à des enfoncements au moins égaux au $1 / 10^{\circ}$ du diamètre du pieu. Lors de l'essai en vraie grandeur, on a pu observer que cette même charge limite réelle $\bar{Q}_{L}$ correspondait à une charge dont le maintien conduisait à alimenter en permanence le vérin. Ce seuil particulier permet souvent d'observer au niveau de la tête de la fondation, des enfoncements de l'ordre de 1 à 5 millimètres par minute.

La valeur de la charge nominale réelle $\bar{Q}_{N}$ a été déterminée en frappant la charge critique de fluage $Q_{F}$, définie selon les prescriptions du Mode Opératoire, d'un coefficient réducteur $F_{s}$ égal à 1,4. On fera remarquer qu'une récente étude de synthèse sur le problème des coefficients de sécurité en matière de fondations profondes [1] a montré que l'adoption de $F_{s}=1,4$ revenait en fait, à prendre en moyenne un coefficient de 2,1 (cas des pieux forés) ou de 1,8 (cas des pieux battus) sur la charge limite réelle $\bar{Q}_{L}$.

\section{Nouvelles règles pressiométriques}

Considérant que le principe même du dimensionnement préconisé par Ménard [12] pouvait être repris pour l'instant, les modifications ou réajustements à apporter aux règles pressiométriques ont essentiellement consisté :

- à redéfinir, en les complétant le cas échéant, les catégories de sols permettant de chiffrer la valeur du facteur de portance $k$,

- à réduire en général mais cela très nettement pour les fondations forées, les valeurs des facteurs $k$,

- à réduire ou à augmenter, suivant le type de sol, le mode de mise en œuvre de la fondation ou la nature de son fût, les valeurs du frottement unitaire $q_{s}$.

\subsection{Nouvelles valeurs du facteur de portance k}

Le choix de ce paramètre s'effectue à partir du tableau 3 et des abaques associées de la figure 7 .

Les valeurs de $\mathrm{k}$, indépendamment de la catégorie de sol, sont rattachées à un type particulier de fondation. On distingue ainsi un premier groupe (I), regroupant les fondations forées et injectées sous faible pression et un second groupe (II), dans lequel entrent les fondations battues, foncées ou injectées sous haute pression. Entrent dans chacun de ces groupes, des fondations dont l'appelation correspond à celle établie par le dernier D. T. U. «Fondations Profondes " [5] (voir annexe).

\section{Groupe I}

- Pieux forés simples,

- pieux forés tubés,

- pieux forés à la boue,

- pieux forés à la tarière creuse,

- micropieux de type I (injectés sous faible pression),

- puits,

- barrettes.

* A base normalement élargie, pas plus de $50 \%$ du diamètre du fût.

**Types pieux injectés métalliques type SOLETANCHE par exemple.

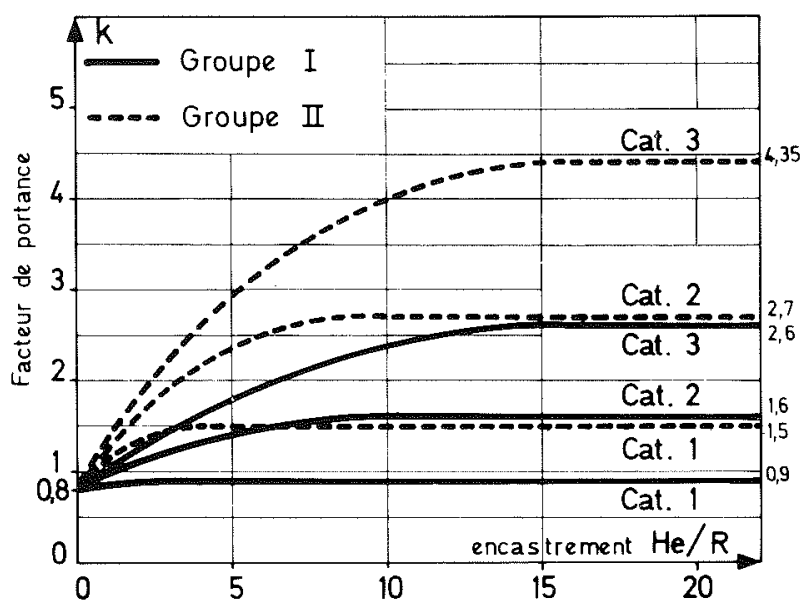

Fig. 7 Nouvelles règles pressiométriques. Abaques pour la détermination du facteur de portance $k$

\section{Groupe II}

- Pieux vissés moulés,

- pieux battus enrobés (injectés sous faible pression),

- pieux battus préfabriqués,

- pieux métalliques battus,

- pieux tubulaires précontraints,

- pieux battus pilonnés*,

- pieux battus moulés,

- pieux en béton foncés,

- pieux en métal foncés,

- micropieux de type II (ou pieux de petits diamètres injectés sous haute pression de diamètre inférieur à $250 \mathrm{~mm}$ ),

- pieux injectés sous haute pression de gros diamètre (par opposition aux micropieux de type II ces pieux regroupent des pieux de fort diamètre, supérieur à $250 \mathrm{~mm})^{\star \star}$.

\begin{tabular}{|c|c|c|}
\hline $\begin{array}{c}\text { Pression limite } \\
p_{e}\left(10^{5} \mathrm{~Pa}\right)\end{array}$ & Nature des sols & Catégorie \\
\hline $\begin{array}{l}<7 \\
<8 \\
<7\end{array}$ & $\begin{array}{l}\text { argile molle } \\
\text { limon + craie molle } \\
\text { sable argileux à limoneux ou } \\
\text { vasard lâche }\end{array}$ & 1 \\
\hline $\begin{array}{l}10 \text { à } 20 \\
12 \text { à } 30 \\
15 \text { à } 40 \\
10 \text { à } 25 \\
25 \text { à } 40 \\
>30 \\
>45\end{array}$ & $\begin{array}{l}\text { sable + grave moyen compacts } \\
\text { argile + limon compacts } \\
\text { marne + marno-calcaire } \\
\text { craie altérée } \\
\text { roche altérée } \\
\text { craie fragmentée } \\
\text { marne très compacte }\end{array}$ & $s$ \\
\hline $\begin{array}{l}>25 \\
>45\end{array}$ & $\begin{array}{l}\text { sable + gravier compacts à très } \\
\text { compacts } \\
\text { roche fragmentée }\end{array}$ & S \\
\hline
\end{tabular}

Tableau 3 Nouvelles règles pressiométriques. Classification des sols en catégories 
On remarquera que le tableau III ne retient plus que trois catégories, contrairement aux anciennes règles qui en comptaient quatre*. Ce même tableau fait apparaître en revanche et nommément, les craies ou les marnes rencontrées fréquemment sur notre territoire. Pour les sols intermédiaires, situés entre 2 plages de pressions limites, on sera conduit à adopter une valeur de $\mathrm{k}$ interpolée.

La comparaison des anciennes et nouvelles abaques déterminant $\mathrm{k}$ (voir fig. $5 \mathrm{a}$ et fig. 7) permet de constater que les nouvelles valeurs du facteur de portance ne représentent plus que $50 \%$ des anciennes valeurs pour les pieux du premier groupe et $75 \%$ pour les pieux du second groupe.

\subsection{Nouvelles valeurs du frottement latéral limite unitaire $\mathrm{q}_{\mathrm{s}}$}

Les nouvelles valeurs de $q_{s}$ sont à calculer à partir des abaques des figures $8 \mathrm{a}$ et $8 \mathrm{~b}$ et du tableau IV associés. Ce dernier, indique en fonction du mode de mise en œuvre du pieu et du matériau constitutif de son fût, de la nature du sol et de sa compacité, l'abaque à prendre en compte au niveau de la prévision de $q_{s}$.

On notera que les abaques $\mathrm{A}, \mathrm{B}$ et $\mathrm{C}$ figurent bien en tant que tels dans les règles Ménard [12]. On leur a donné toutefois ici, une signification quelque peu nouvelle. On verra brièvement et dans l'ordre, les modifications effectuées ou extensions apportées.

\section{Abaque $\mathrm{A}$}

Cet abaque a été essentiellement rattaché à une classification des sols plus affinée, intégrant les craies. Par rapport aux anciennes règles, les abattements à effectuer sur les valeurs $q_{s}$ ont été parfois accrus: $60 \%$ au lieu de 25 à $50 \%$ dans certains cas. L'abaque dérivé $\mathrm{Abis}$ permet de chiffrer les différents taux d'abattement.

\section{Abaque $\mathrm{B}$}

Cet abaque est maintenant proposé pour chiffrer les valeurs de $q_{s}$ à prendre en compte pour toutes les parties courantes du fût et non comme cela était le cas dans les anciennes règles sur la partie de fût située à proximité immédiate de la pointe. L'utilisation de cet abaque a été également étendue au cas des pieux forés (dans les craies ou marnes) et les pieux battus aciers (dans les craies et sables ou graviers).

\section{Abaque $\mathrm{C}$}

Cet abaque qui ne s'appliquait selon les règles Ménard qu'aux pieux dilatés est maintenu et son application, étendue aux pieux injectés sous faible pression ainsi qu'aux pieux forés dans les marnes et marno-calcaires.

\section{Abaque D}

Celui-ci est nouveau et s'applique aux pieux injectés sous haute pression mis en œuvre dans les argiles, limons, sables, graves et craies.

\section{Abaque $E$ et $F$}

Ceux-ci sont réservés aux marnes très compactes et rochers altérés à fragmentés.
Point important, le tableau IV propose parfois 2 valeurs différentes de $q_{s}$ : une première valeur «plancher» et une seconde valeur, figurant entre parenthèses. La seconde valeur, plus élevée, correspond à des frottements unitaires limites qu'il est possible de mobiliser $^{\star \star}$, à condition de soigner au maximum l'exécution du pieu et de choisir la technique de mise en cuvre la plus performante. Ainsi, pour les chantiers importants, où l'on envisage la confection d'un nombre important de pieux, il est vivement conseillé de vérifier expérimentalement, par un ou plusieurs essais préalables de chargement en vraie grandeur, s'il n'est pas possible de retenir la valeur maximale de $q_{s}$. Il est bien évident que ces essais ne pourront être entrepris que si l'on peut prouver que leur coût sera largement compensé par l'économie réalisée au niveau du dimensionnement.

Le tableau 4 effectue un découpage au niveau du type de pieu et du matériau constitutif de son fût, distinguant ainsi 7 sous-ensembles, Chacun d'eux regroupe un certain nombre de fondations qui entrent dans la classification du D. T.U. «Fondations profondes $\gg$ [5] (voir annexe).

\section{Pieux forés avec fût béton}

- Pieux forés simples,

- pieux forés à la boue,

- pieux forés à la tarière creuse,

- pieux vissés moulés,

- micropieux du type I,

- puits,

- barrette.

\section{Pieux forés tubés avec fût béton ou métal}

- Pieux forés tubés,

- pieux battus moulés.

\section{Pieux battus avec fût béton ou fût métal}

- Pieux battus préfabriqués,

— pieux métalliques battus,

- pieux tubulaires précontraints,

- pieux en béton foncé,

- pieux en métal foncé.

Pieux injecté sous faible pression

- Pieux battus enrobés,

- pieux battus pilonnés.

\section{Pieux injectés sous haute pression}

- Pieux injectés sous haute pression de diamètre supérieur à $250 \mathrm{~mm}$,

- micropieux du type 11.

Dans le cas où l'on doit recourir à plusieurs techniques de forage, c'est-à-dire tuber la partie haute (remblais ou terrains s'éboulant naturellement en raison d'un manque de cohésion ou sous l'action de nappes ou poches d'eau) puis poursuivre sans tubage ou forer sous boue, on appliquera à chacune des parties du fût, la valeur de $q_{s}$ correspondant à la technique utilisée. 

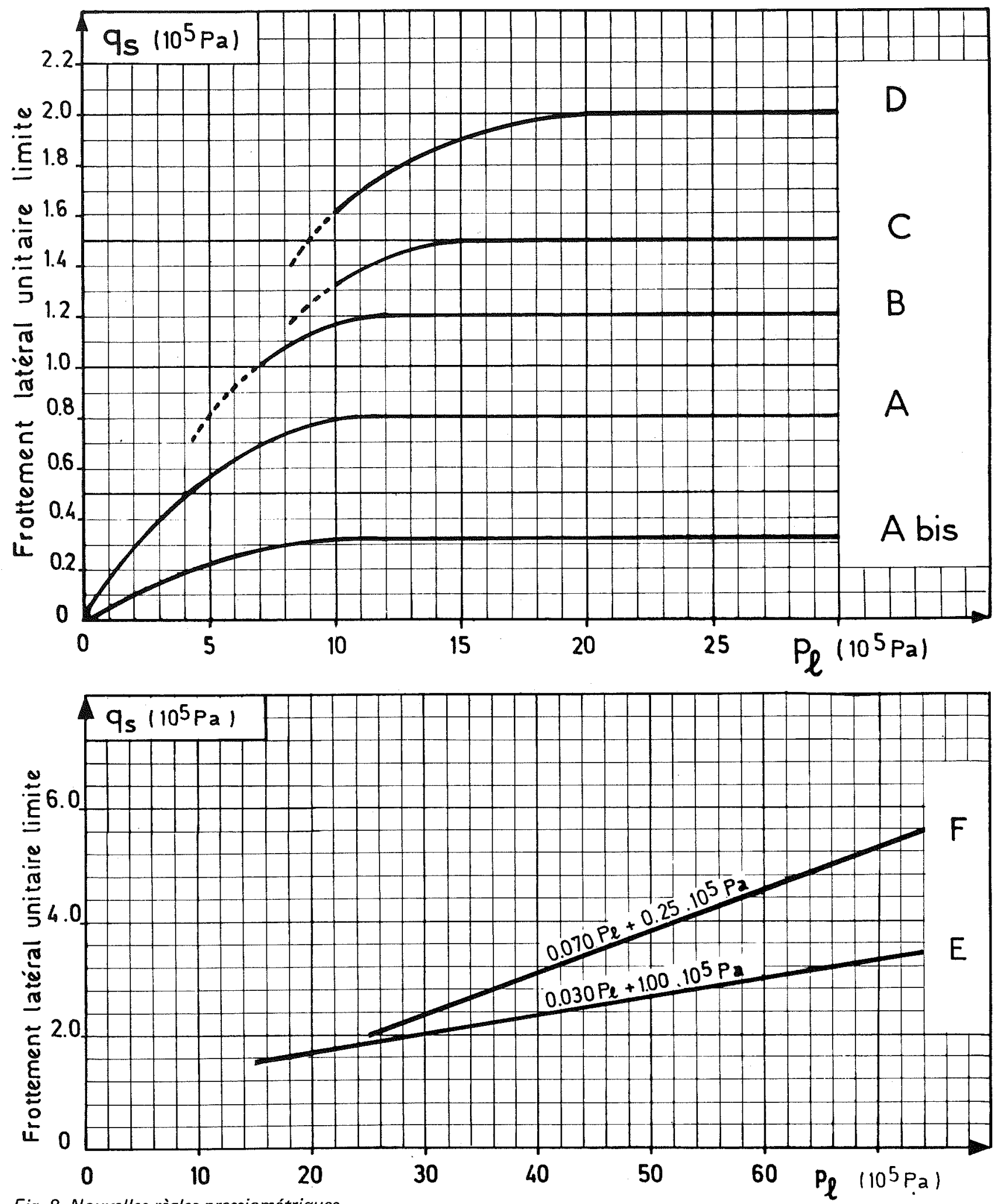

Fig. 8 Nouvelles règles pressiométriques

a) abaques pour la détermination du frottement unitaire $q_{s}$ (argiles, limons, sables, graves, craie, marnes, sols organiques)

b) abaques pour la détermination du frottement unitaire $q_{s}$ (marnes très compacts, rochers)

Pour les pieux à géométrie ou de mise en œuvre particulières, il est recommandé, lors de l'application des abaques de la figure 7 et 8 :

- de considérer pour un profil $\mathrm{H}$ que la surface latérale de celui-ci est déterminée par le périmètre circonscrit,

- de ne tenir compte, sauf vérification expérimentale pour les pieux lancés, d'aucun frottement sur toute la hauteur du fût lancée,
- pour les pieux tubulaires ouverts ${ }^{\star}$ avec formation d'un bouchon en cours de battage, de ne tenir compte, lors des calculs, que de la surface extérieure du pieu,

- pour les pieux injectés sous faible pression ou haute pression, on retiendra comme surface latérale la surface définie par le périmètre de forage,

* Il s'agit là d'un type de pieu assez fréquemment utilisé à l'étranger mais que nous n'avons pas eu l'occasion pour l'instant d'étudier expérimentalement. 


\begin{tabular}{|c|c|c|c|c|c|c|c|c|}
\hline \multirow{3}{*}{$\begin{array}{l}\text { Type } \\
\text { de } \\
\text { sols }\end{array}$} & \multirow{3}{*}{$\begin{array}{l}\text { Pression } \\
\text { limite } \\
p \\
\left(10^{5} \mathrm{~Pa}\right)\end{array}$} & \multicolumn{7}{|c|}{ Mise en œuvre et nature du fût } \\
\hline & & \multirow{2}{*}{\begin{tabular}{|c|} 
Foré \\
Fût béton
\end{tabular}} & \multicolumn{2}{|c|}{ Foré tubé } & \multicolumn{2}{|c|}{ Battu } & \multicolumn{2}{|c|}{ Injecté } \\
\hline & & & Fût béton & Fût métal & Fût béton & Fût métal & $\begin{array}{c}\text { Faible } \\
\text { pression }\end{array}$ & $\begin{array}{l}\text { Haute } \\
\text { pression }\end{array}$ \\
\hline $\begin{array}{l}\text { Sable ar- } \\
\text { gileux à } \\
\text { limoneux } \\
\text { ou vasard }\end{array}$ & $<7$ & A bis & A bis & A bis & A bis & A bis & A & - \\
\hline $\begin{array}{l}\text { Graie } \\
\text { molle }\end{array}$ & $<7$ & A bis & A bis & A bis & A bis & A bis & A & - \\
\hline $\begin{array}{c}\text { Argile } \\
\text { molle à } \\
\text { compacte }\end{array}$ & $\leqslant 30$ & $\begin{array}{l}(A)^{*} \\
A \text { bis }\end{array}$ & $\begin{array}{l}(A)^{*} \\
A \text { bis }\end{array}$ & Abis & $\begin{array}{l}(A)^{*} \\
A \text { bis }\end{array}$ & A bis & A & $\mathrm{D}^{* *}$ \\
\hline $\begin{array}{l}\text { Limon à } \\
\text { limon } \\
\text { compact }\end{array}$ & $\leqslant 30$ & $\begin{array}{l}(A)^{*} \\
A \text { bis }\end{array}$ & $\begin{array}{l}(A)^{*} \\
\text { A bis }\end{array}$ & A bis & $\begin{array}{l}(A)^{\star} \\
A \text { bis }\end{array}$ & A bis & A & $D^{* *}$ \\
\hline $\begin{array}{l}\text { Sables et } \\
\text { graves } \\
\text { moyennement } \\
\text { compacts }\end{array}$ & 10 à 20 & $\begin{array}{c}(B)^{\star} \\
A\end{array}$ & $\begin{array}{l}(A)^{*} \\
\text { A bis }\end{array}$ & A bis & $\begin{array}{c}(B)^{*} \\
A\end{array}$ & A & $B$ & $\geqslant D$ \\
\hline $\begin{array}{c}\text { Sables et } \\
\text { grave compacts } \\
\text { à très } \\
\text { compacts }\end{array}$ & $>25$ & $\begin{array}{l}(\mathrm{C})^{*} \\
\mathrm{~B}\end{array}$ & $\begin{array}{c}(B)^{*} \\
A\end{array}$ & A & $\begin{array}{c}(\mathrm{C})^{*} \\
\mathrm{~B}\end{array}$ & B & $\mathrm{C}$ & $\geqslant D$ \\
\hline $\begin{array}{l}\text { Craie al- } \\
\text { térée à } \\
\text { fragmentée }\end{array}$ & $>10$ & $\begin{array}{l}(\mathrm{C})^{*} \\
\mathrm{~B}\end{array}$ & $\begin{array}{c}(B)^{*} \\
A\end{array}$ & A & $\begin{array}{c}(C)^{*} \\
B\end{array}$ & B & $\mathrm{C}$ & $\geqslant D$ \\
\hline $\begin{array}{l}\text { Marne et } \\
\text { marno- } \\
\text { calcaire }\end{array}$ & 15 à 40 & $\begin{array}{l}(E)^{\star} \\
C\end{array}$ & $\begin{array}{c}(\mathrm{C})^{*} \\
\mathrm{~B}\end{array}$ & B & ${ }^{\star \star \star} E$ & ${ }^{* * *} E$ & $E$ & $\mathrm{~F}$ \\
\hline $\begin{array}{l}\text { Marne très } \\
\text { compacte }\end{array}$ & $>45$ & $E$ & - & - & - & - & $\mathrm{F}$ & $>F$ \\
\hline $\begin{array}{l}\text { Rocher } \\
\text { altéré }\end{array}$ & 25 à 40 & $\mathrm{~F}$ & $\mathrm{~F}$ & - & $\star \star \star * F$ & $\star \star \star F$ & $\geqslant F$ & $>F$ \\
\hline $\begin{array}{l}\text { Rocher } \\
\text { fragmenté }\end{array}$ & $>45$ & $\mathrm{~F}$ & - & - & - & - & $\geqslant F$ & $>F$ \\
\hline
\end{tabular}

* Les valeurs entre parenthèses ( ) correspondent pour les pieux forés à une exécution soignée du pieu et une technologie de mise en cuvre susceptible de remanier au minimum le sol au contact du fût mais pour les pieux battus à un resserrement du sol sur le pieu.

** Préconisé pour des sols dont $\mathrm{p}_{\ell}>15 \cdot 10^{5} \mathrm{~Pa}$.

*** Seulement pour les cas où le battage est possible.

Tableau 4 Nouvelles règles pressiométriques. Tableau pour le choix des abaques relatifs au frottement unitaire $q_{s}$

- pour les sols très compacts $\left(\mathrm{p}_{\ell}>2,5 \mathrm{MPa}\right)$, les argiles raides, les marnes ou le rocher altéré, on calculera la valeur de $\mathrm{q}_{\mathrm{s}}$ à partir des valeurs de pression limite $p_{\ell}$ du terrain effectivement mesurées.
L'utilisation d'un pressiomètre type « rocher » est donc recommandée,

- enfin, il ne sera pas tenu compte du frottement latéral sur le premier mètre du fût. 


\subsection{Paramètres calculés et paramètres mesurés} [15] [16] [17] [18] [19] [20] [21] [22] [23]

L'interprétation de l'ensemble des résultats obtenus après essais de chargement a permis de déduire, entre autres, les valeurs réelles $\bar{k}$ des facteurs de portance et des frottements latéraux unitaires $\bar{q}_{s}$. Elles ont été reportées sur les figures 9 à 10 qui les situent également par rapport aux abaques correspondants, proposés par les nouvelles règles. Ces figures regroupent un total de 23 sites qui sont les seuls, sur lesquels la charge limite $\bar{Q}_{L}$ ait été effectivement atteinte. En ce qui concerne le facteur de portance, la catégorie du sol $n^{\circ} 2$, contrairement aux catégories $\mathrm{n}^{\text {os }} 1$ et 3 , est de loin la mieux représentée. Elle englobe des sols que leur compacité incite à retenir comme couche d'encastrement de la pointe mais que l'on retrouve aussi le plus souvent sur l'ensemble de notre territoire.

Les figures $9 a, b, c$ permettent de constater que pour l'ensemble des trois catégories, les facteurs de portance mesurés $k$ sont inférieurs aux valeurs préconisées par les anciennes règles (voir fig. $5 \mathrm{a}$ ). Cette remarque est particulièrement vraie pour les pieux forés. Les valeurs les plus faibles de $\bar{k}$, se rapportent à des pieux dont la mise en cuvre a remanié le fond du forage ou pour lesquels ce dernier n'a pas fait l'objet d'un curage suffisamment soigné. Les valeurs correspondant aux cas les plus critiques comportent l'indice re. Par contre l'indice i correspond à des sols que leur pression limite situe entre deux catégories.

Les figures $10 \mathrm{a}$ à $10 \mathrm{~g}$ font apparaître, en regard des nouveaux abaques proposés, les valeurs mesurées $\bar{q}_{s}$ du frottement unitaire, sur un total de 25 sites et cela pour le premier chargement uniquement.

\section{Remarques sur la validité des nouvelles règles}

II y a lieu de faire remarquer en premier lieu, que les règles proposées, en dépit du fait qu'elles soient étalonnées sur des essais réels, ne suffiront pas à garantir dans tous les cas une portance réelle de l'ordre de celle estimée par le calcul. Le projeteur sera toujours à la merci d'un remaniement plus ou moins intense des parois du forage [6] ou, cas malheureusement trop fréquent pour les pieux forés, d'un contact pointe-sol, de qualité très médiocre (décompression du fond ou curage insuffisant [24] [25]. En fait, il est toujours aussi difficile de faire refléter à des règles de calcul fiable, l'incidence fondamentale de la mise en œuvre et de ses aléas, sur la portance finale d'un pieu. II s'agit probablement là de l'un des problèmes le plus difficile à appréhender aujourd'hui et à répercuter sur les méthodes de calcul des pieux [26].

II est intéressant, dans le but de mieux faire saisir l'incidence du réajustement des paramètres $k$ et $q_{s}$ sur la portance prévisionnelle d'un pieu, de donner un exemple concret assez caractéristique. Celui-ci intéresse 2 pieux forés à la tarière, sur les 11 premiers mètres environ à l'abri d'un tubage provisoire, par la suite sans tubage ni boue, c'est-à-dire à sec. La figure 11 synthétise le contexte géotechnique et situe par rapport à ce dernier les 2 pieux, lesquels, après avoir été équipés d'extensomètres amovibles, ont été soumis à des essais statiques de chargement.

Le tableau 5 récapitule les données des essais et des calculs effectués à partir des anciennes règles pressiométriques et des nouvelles.

II y a lieu de rappeler les hypothèses principales du calcul. Elles figurent au tableau 6, les valeurs données entre parenthèses correspondant aux anciennes règles.

\begin{tabular}{|c|c|c|c|c|c|c|}
\hline $\begin{array}{c}\text { Pieu } \\
n^{\circ}\end{array}$ & $\begin{array}{c}\text { moyen } \\
\text { d'estimation }\end{array}$ & $\begin{array}{c}\text { Charges limites } \\
Q_{L}=Q_{L}^{F}+Q_{L}^{P} \\
(k N)\end{array}$ & $\begin{array}{c}\text { Charge nominale } \\
Q_{N} \\
(k N)\end{array}$ & $\begin{array}{c}\text { Charge critique } \\
Q_{F} \\
(k N)\end{array}$ & $\frac{\overline{Q_{L}}}{Q_{N}}$ & $\frac{Q_{F}}{Q_{N}}$ \\
\hline \multirow{3}{*}{$\begin{array}{c}1 \\
(\varnothing 680 / 620)\end{array}$} & essai de pieu & $2400=2190+250$ & 1210 & 1700 & 2,02 & 1,40 \\
\hline & $\begin{array}{l}\text { nouvelles } \\
\text { règles }\end{array}$ & $2350=2140+210$ & 1140 & - & 2,14 & 1,49 \\
\hline & $\begin{array}{l}\text { anciennes } \\
\text { règles }\end{array}$ & $3220=2920+300$ & 1560 & - & 1,56 & 1,09 \\
\hline \multirow{3}{*}{$\begin{array}{c}2 \\
(\varnothing 880 / 700)\end{array}$} & essai de pieu & $5400=4020+1380$ & 2780 & 3900 & 1,94 & 1,40 \\
\hline & $\begin{array}{l}\text { nouvelles } \\
\text { règles }\end{array}$ & $5440=4140+1300$ & 2500 & - & 2,16 & 1,56 \\
\hline & $\begin{array}{l}\text { anciennes } \\
\text { règles }\end{array}$ & $7880=5270+2610$ & 3500 & - & 1,54 & 1,11 \\
\hline
\end{tabular}

Tableau 5 

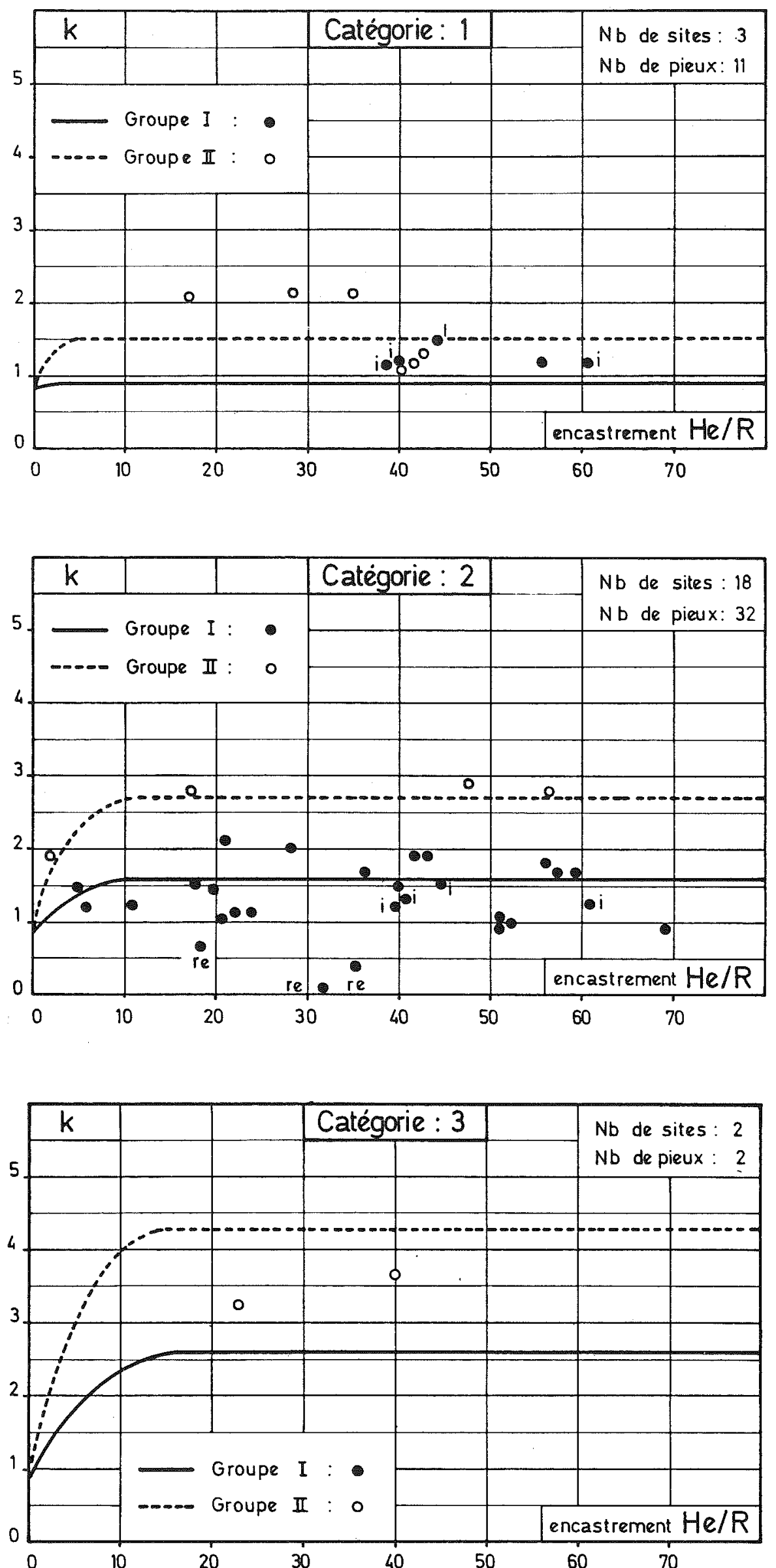

Fig. 9 Valeurs du facteur de portance $k$ mesurées sur différents sites pour des pieux relevant des différentes catégories a) catégorie 1

b) catégorie 2

c) catégorie 3 

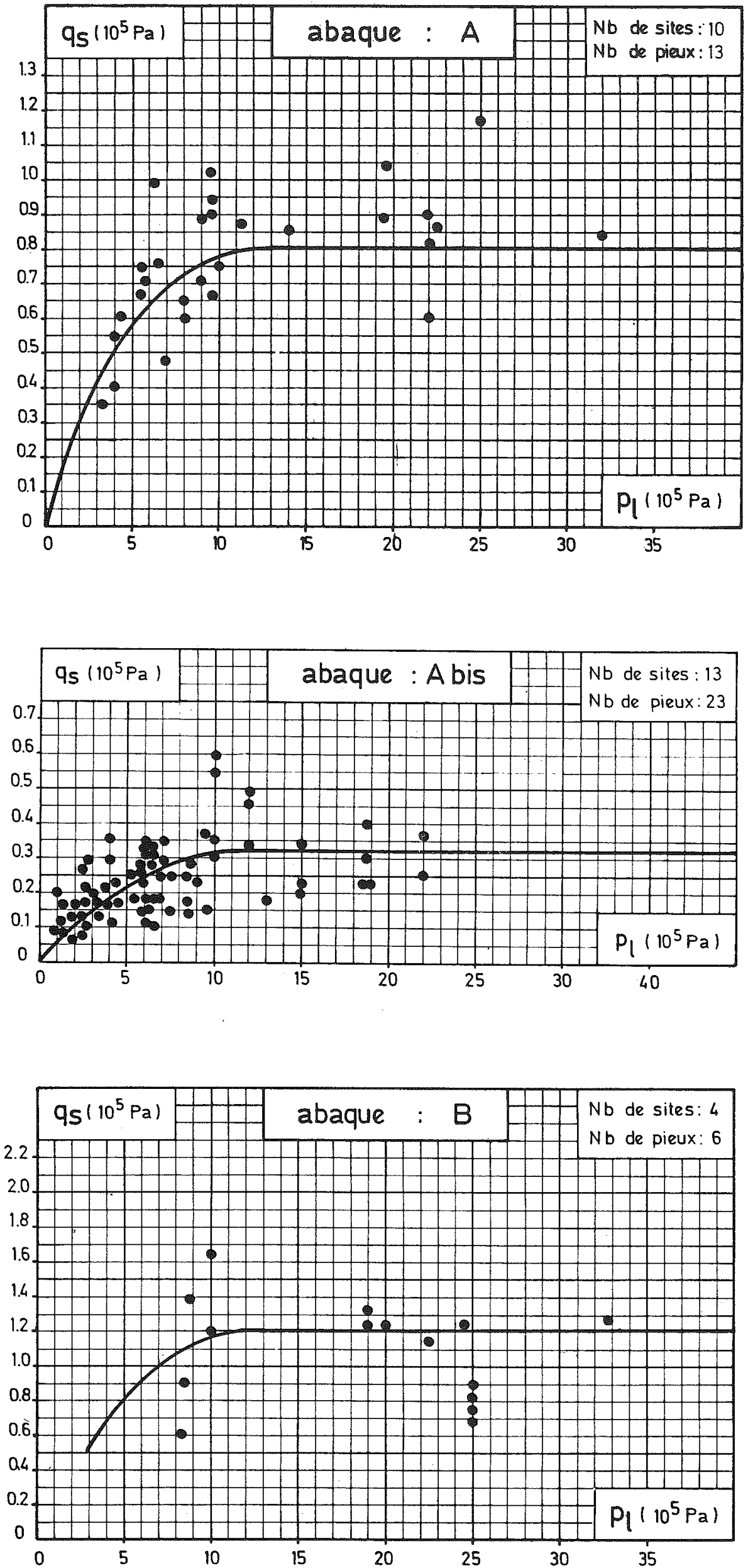

Fig. 10 Valeurs du frottement latéral unitaire $q_{s}$ mesurées sur différents sites 

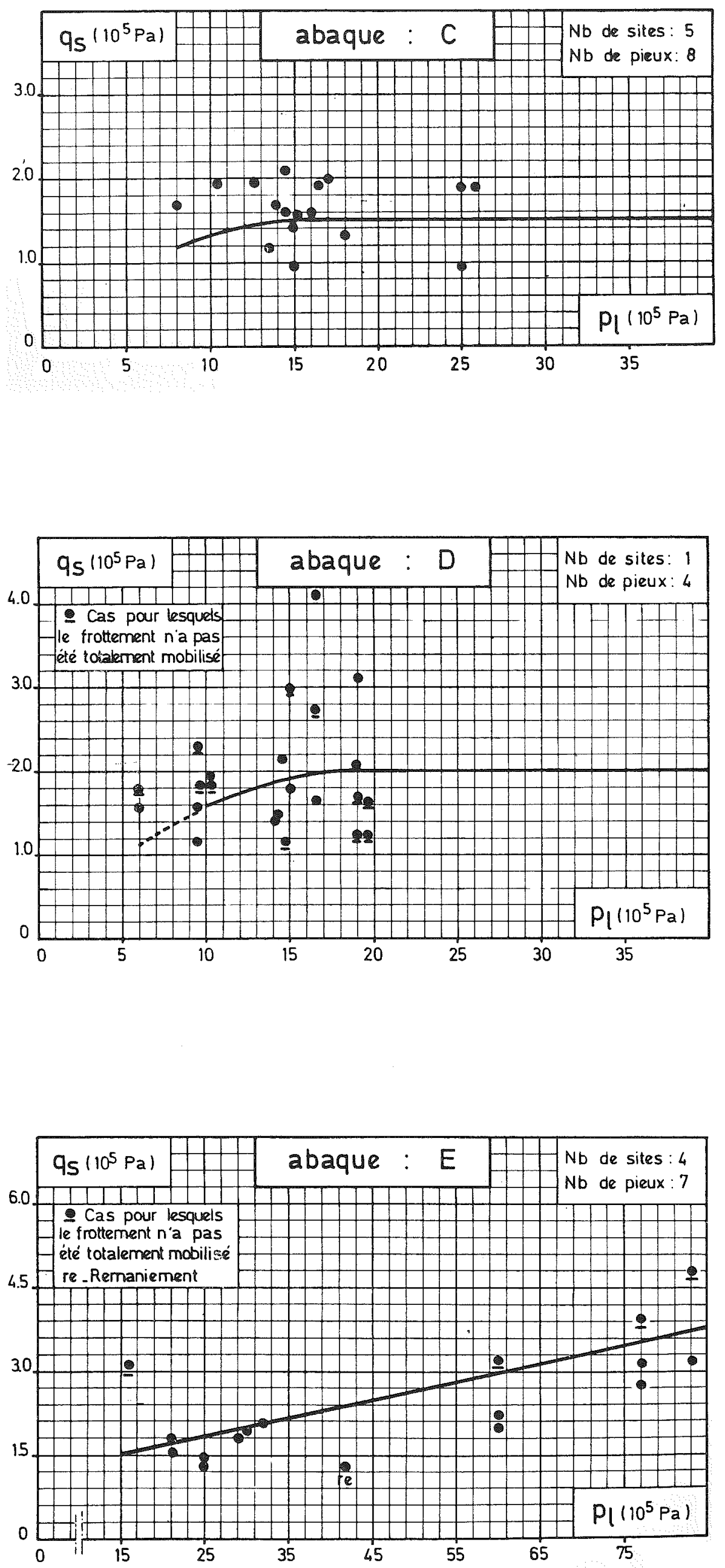


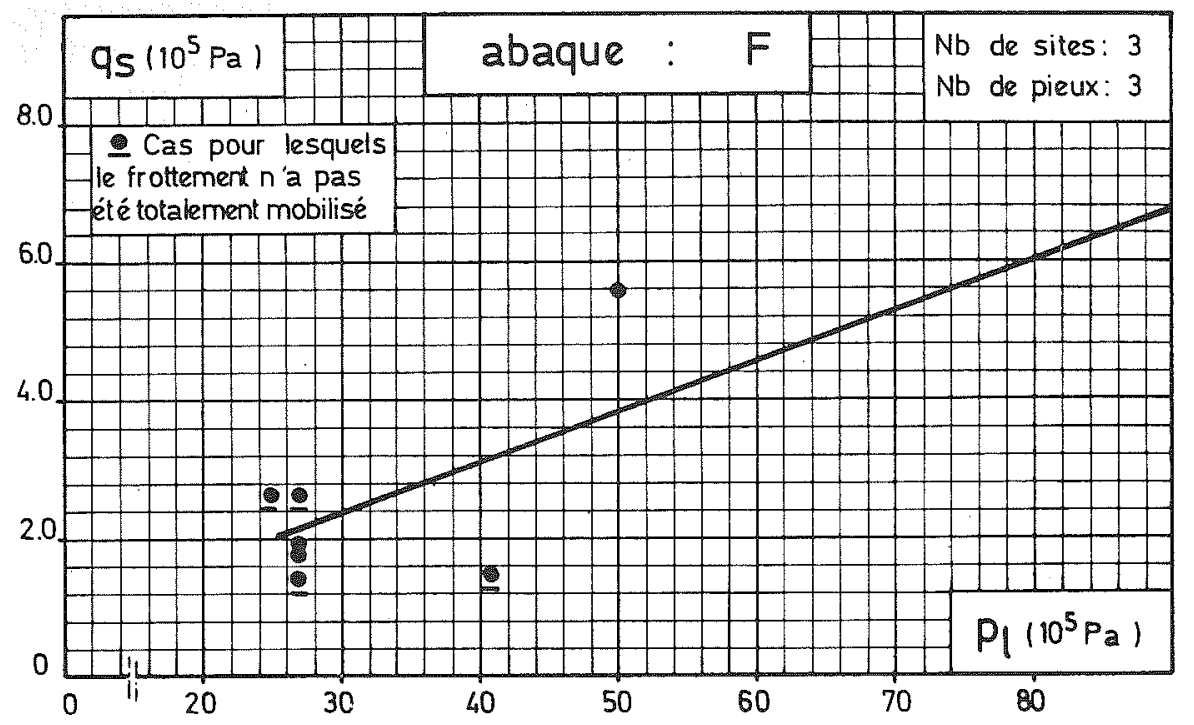

\begin{tabular}{c|c|c|c|c|c}
\hline Pieu & $\begin{array}{c}\text { Facteur de portance } \\
k\end{array}$ & Catégorie de sol & $\begin{array}{c}\text { Encastrement relatif } \\
\text { he/R }\end{array}$ & \multicolumn{2}{|c}{$\begin{array}{c}\text { Frottement unitaire } \mathrm{q}_{\mathrm{s}} \\
\left(\mathrm{kN} / \mathrm{m}^{2}\right)\end{array}$} \\
\cline { 5 - 6 } & & $1 / 2$ & 56 & 25 & 80 \\
\hline 1 & 1,28 & $(1)$ & & $(60)$ & $(80)$ \\
\hline & $(1,8)$ & 2 & 59 & 25 & 80 \\
& 1,6 & $(2)$ & & $(60)$ & $(80)$ \\
\hline
\end{tabular}

Tableau 6

ROUBAIX . Hopital (1980)

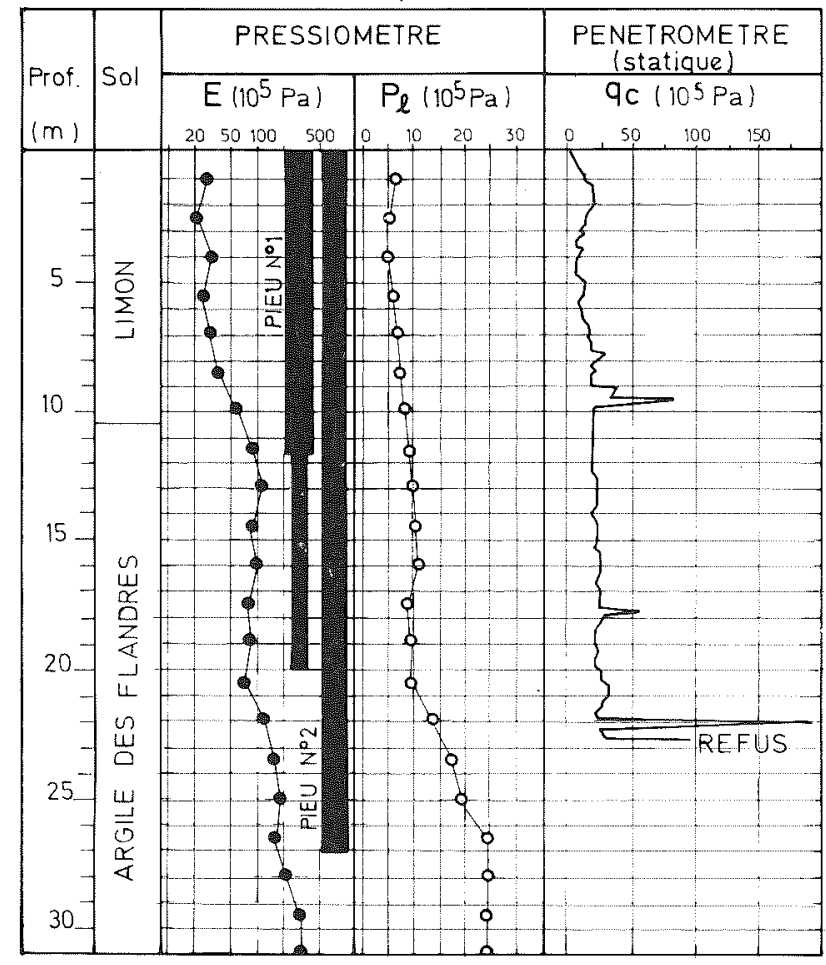

Fig. 11 Coupe géotechnique des sols sur le site de Roubaix
L'examen du tableau $V$ montre bien que l'application des nouvelles règles conduit à des portances prévisionnelles beaucoup mieux calées sur la réalité. On aura remarqué l'extrême faiblesse des coefficients de sécurité réels auxquels conduisent, dans le cas évoqué, les anciennes règles. Le coefficient de sécurité, par rapport à la charge critique de fluage $Q_{F}$ (seuil critique au-delà duquel les enfoncements sous charge constante sont non seulement importants mais risquent de croître dans le temps) reste dangereusement bas : 1,09 et 1,11 pour le premier et second pieu. Lorsque l'on sait de plus, que les deux pieux essayés ont fait l'objet d'une exécution particulièrement soignée (curage parfait du fond de forage, décapage des parois), on comprend que des pieux réalisés dans des conditions de chantier habituelles risquent d'accuser, dimensionnés selon les anciennes règles, de graves défauts de portance.

Il faut toutefois bien faire remarquer, que-si dans l'exemple exposé, l'application des nouvelles règles conduit à limiter la portance nominale des pieux, dans d'autres cas, où l'on est en présence de sols de natures différentes mais aussi, où l'on aura pu recourir à d'autres techniques de mise en œuvre, ces mêmes règles permettront d'augmenter sensiblement la portance nominale. La figure 12, qui présente sous forme d'histogrammes (établis uniquement pour les 32 sites sur lesquels ont opéré les Laboratoires des Ponts et 


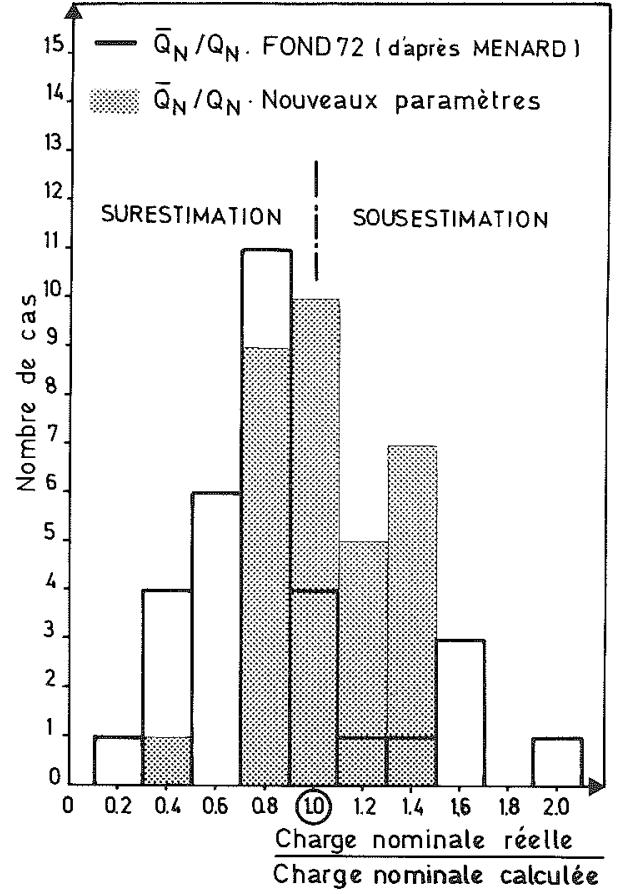

Fig. 12 Histogramme relatif aux rapports des charges nominales réelles sur charges nominales calculées

Chaussées) des rapports de portances nominales, démontre clairement que la prise en compte des nouveaux paramètres $k$ et $q_{s}$, ne provoque pas le sous-dimensionnement systématique des fondations. On constate par contre, un meilleur alignement général des portances nominales calculées sur les portances correspondantes réelles. La figure 13 enfin, qui représente l'histogramme des rapports des termes de points $\mathrm{Q}_{\mathrm{L}}^{\mathrm{P}}$, illustre bien la surestimation très marquée en ce terme, par les anciennes règles.

\section{L'amplitude des tassements réels. Validité des méthodes de calcul}

Après calcul de la portance nominale d'une fondation profonde, le praticien est parfois conduit à s'interroger sur l'importance des tassements (ou enfoncements) que celle-ci accusera sous cette même charge. Il existe plusieurs méthodes pour effectuer ce calcul mais restant ici dans le cadre des règles pressiométriques, on ne traitera, très brièvement, que des 2 méthodes qui leur sont associées et qui ont été proposées par Gambin et Cassan [27] [28]. Et cela uniquement pour les situer par rapport aux tassements réellement mesurés.

II faut présenter avant toutes choses, les valeurs des enfoncements globaux $y_{N}$, effectivement mesurés en tête des fondations. On entend par «enfoncement global » la somme des déplacements instantanés et différés* et des déformations des fûts sous la charge. La figure 14 donne ces enfoncements globaux, pour 56 essais de chargement choisis parmi les plus représentatifs, effectués sur 26 sites et intéressant des pieux forés, battus et injectés. On peut constater que sur l'ensemble de ces essais:

- 37 indiquaient $\quad y_{N}<5 \mathrm{~mm}$, soit $\approx 66 \%$ du total

-12 indiquaient $5<\mathrm{y}_{\mathrm{N}}<10 \mathrm{~mm}$, soit $=21 \%$ du total

- 2 indiquaient $10<\mathrm{y}_{\mathrm{N}}<15 \mathrm{~mm}$, soit $=4 \%$ du total

*En raison du mode particulier de chargement par paliers successifs.

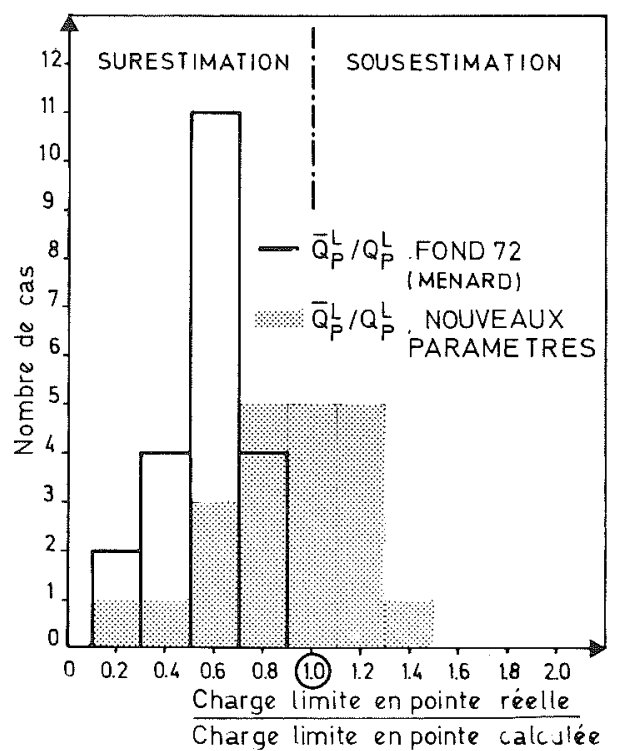

Fig. 13 Histogramme des charges en pointe $Q_{L}{ }^{P}$ calculées et réelles

- 3 indiquaient $15<\mathrm{y}_{\mathrm{N}}<20 \mathrm{~mm}$, soit $\simeq 5 \%$ du total - 2 indiquaient $20<\mathrm{y}_{\mathrm{N}}<25 \mathrm{~mm}$, soit $\simeq 4 \% \mathrm{du}$ total.

On voit donc que dans la quasi-totalité des cas, les enfoncements globaux $y_{N}$ sous charge nominale réelle $\bar{Q}_{N}$ sont très faibles: 0,2 à $2 \%$ du diamètre si l'on raisonne en enfoncements relatifs $y_{N} / D$. II faut souligner aussi la dispersion des enfoncements mesurés. II est intéressant de faire remarquer enfin, qu'il ne semble exister apparemment aucune relation entre ces valeurs et la nature des sols ou le type de mise en cuvre.

En ce qui concerne la validité des méthodes proposées par Gambin et Cassan, on a effectué une comparaison des enfoncements calculés à partir de ces dernières et des enfoncements effectivement mesurés. Les résultats de cette comparaison sont illustrés par 4 exemples types (fig. 15 à $15 \mathrm{~d}$ ), intéressant des pieux non seulement convenablement exécutés mais pour lesquels on possédait la totalité des paramètres requis par les méthodes proposées*. Les courbes d'enfoncement réelles ${ }^{* *}$ relevées sur chantier et qui sont rapportées sur les figures 15 a et $15 \mathrm{~d}$ montrent, que si l'amplitude des tassements (ou enfoncements) calculés, ne correspondent pas exactement, les ordres de grandeur sont bien les mêmes. Aussi, compte-tenu des grandeurs en jeu, inférieures dans la plupart des cas au centimètre, on peut considérer que les 2 méthodes de calcul évoquées conduisent à des valeurs de tassement sous charge nominale $Q_{N}$ parfaitement acceptables pour la pratique courante. En outre, et là l'argument est de poids, les méthodes de Gambin et Cassan ont l'avantage de reposer sur un essai de sol qui, comme nous l'avons $v u$, est pratiquement réalisable et représentatif dans toutes les configurations de sols.

*En particulier les modules alternés du sol $E_{a}$ pour les pieux battus, ainsi que les modules d'élasticité du matériau constitutif des fûts.

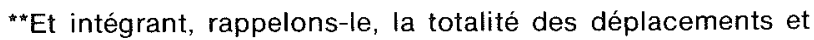
déformations subies par le pieu. 


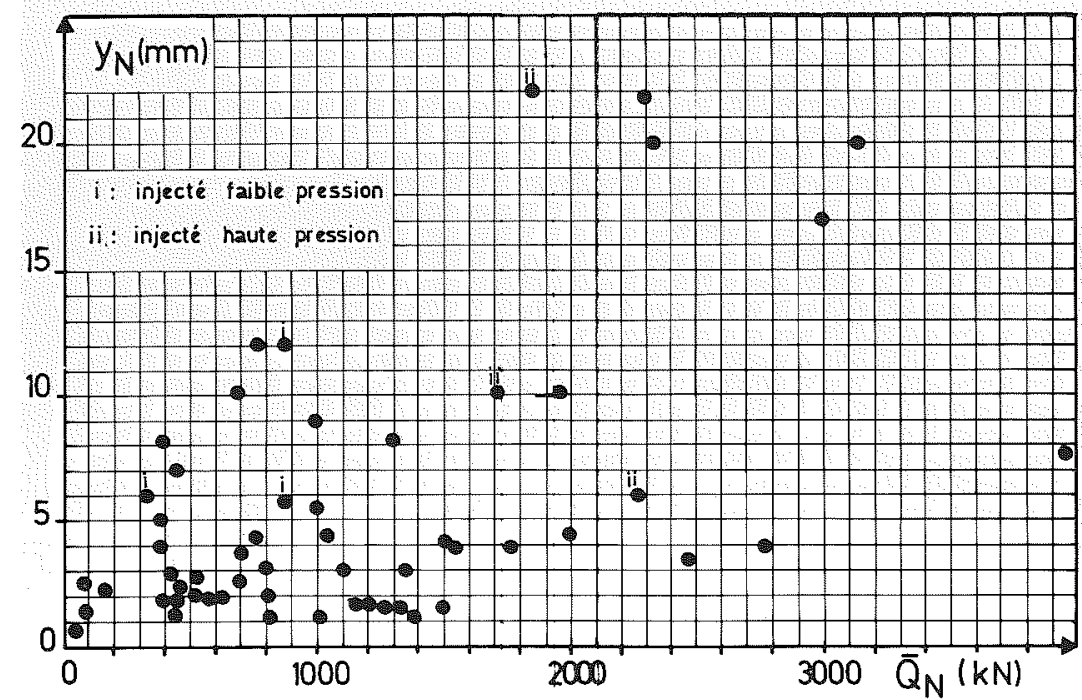

Fig. 14 Enfoncements globaux $y_{N}$ sous les charges nominales réelles pour différents sites
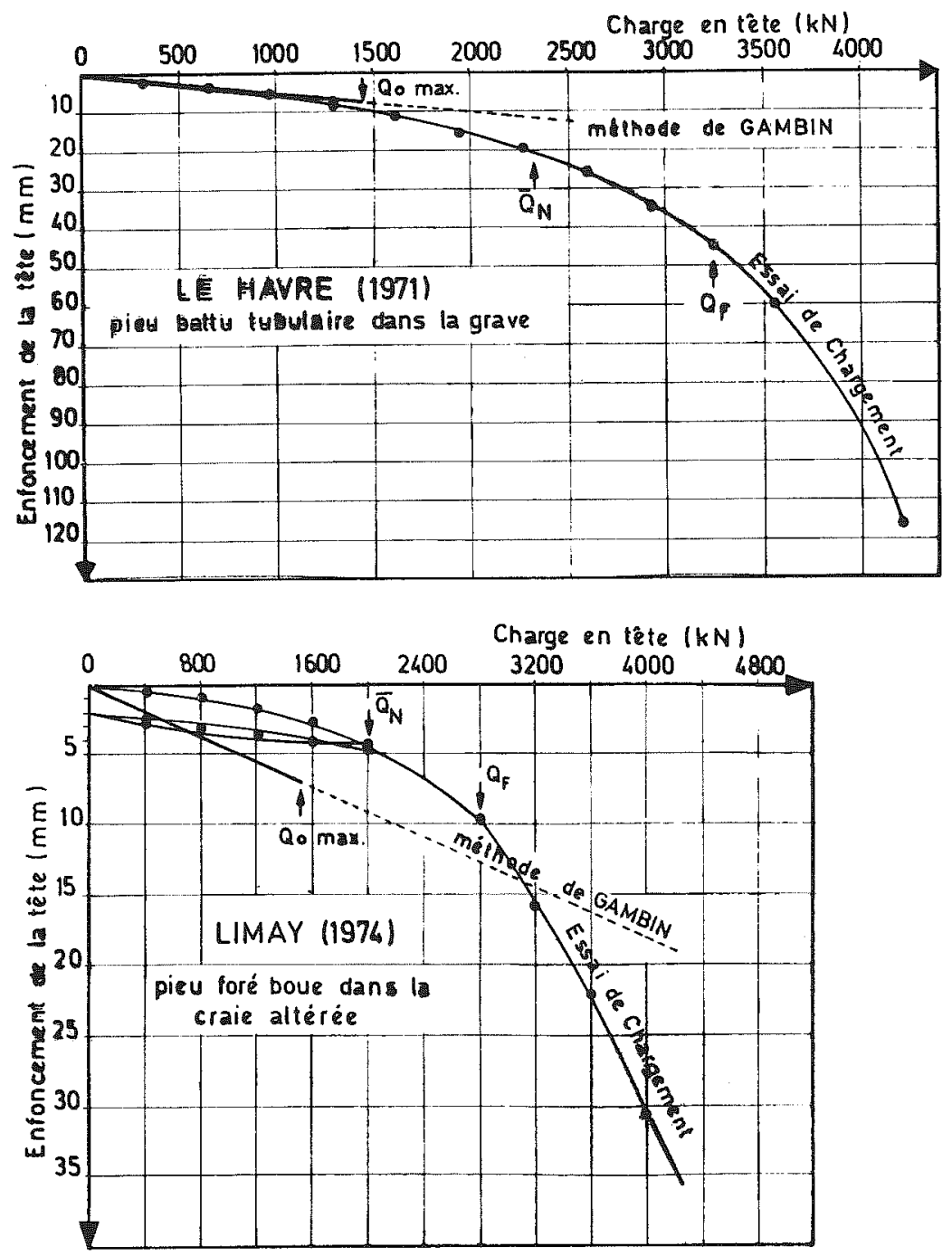

Fig. 15 Courbes des enfoncements globaux réels et calculés d'après les méthodes Gambin et Cassan a) site du Havre

b) site de Limay

c) site de Fieury-sur-Andelle

d) site de Saint-Denis 

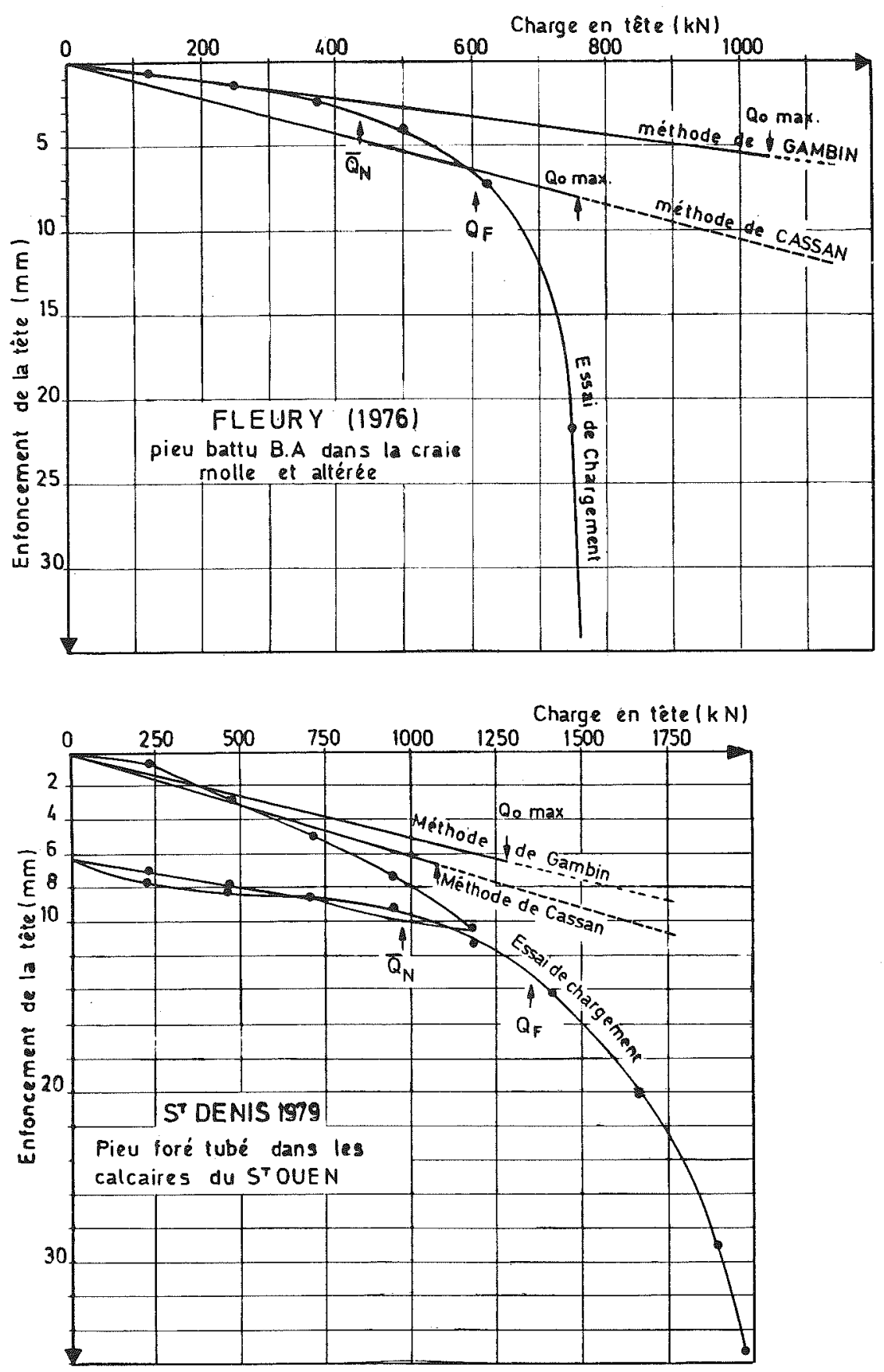

\section{Conclusions}

L'étude de synthèse sur la portance des fondations profondes, effectuée à partir des résultats obtenus après réalisation d'un nombre très important d'essais de chargement en vraie grandeur, a permis de vérifier que la méthode pressiométrique énoncée par Ménard s'avérait être, au dépit de certaines insuffisances, l'une des plus fiables.

Présentant en outre l'avantage capital de reposer sur un essai, réalisable dans pratiquement tous les types de sols, cette méthode et les règles qui lui étaient associées, méritaient d'être affinées et mieux calées sur la réalité.

Considérant aussi, que le principe même du dimensionnement préconisé par les règles Ménard pouvait être retenu, la remise à jour de celles-ci a principalement consisté à établir de nouveaux abaques donnant de nouvelles valeurs des facteurs de portance $k$ (calcul de la résistance de pointe) et du frottement latéral unitaire $\mathrm{q}_{\mathrm{s}}$.
Les conditions d'application de ces abaques sont définies au paragraphe 6 du présent article. On trouvera également dans ce même paragraphe et le suivant, une comparaison des valeurs caractéristiques $k$ et $q_{s}$ mesurées et préconisées par les nouvelles règles. Ces dernières s'appliquent à la quasi totalité des fondations profondes actuellement mises en œuvre sur le marché. On fera remarquer que dans le but de faciliter la tâche des projeteurs, ces nouvelles règles au niveau de la classification des fondations, ont été alignées sur le dernier D.T.U. 13.2 «Fondations Profondes".

Enfin, en ce qui concerne l'évaluation des tassements d'une fondation profonde, on a pu testé la validité des méthodes proposées par Gambin et Cassan, basée sur l'utilisation des paramètres pressiométriques (module $E$ et pression limite $p_{\ell}$ ). On a vérifié expérimentalement que ces 2 méthodes conduisaient à des ordres de grandeur des tassements calculés, acceptables dans la pratique courante. 


\section{Références bibliographiques}

[1] M. Bustamante, L. Gianeselli. Capacité portante des pieux isolés sous charge statique. Rapport de recherche interne 1.05.02.6. LCPC. Décembre 1978 et 1979.

[2] Jelinek, Korek, Stocker. Load tests on 5 large diameter Bored piles in clay. IX Congrès de Tokyo 1977.

[3] Van Wambeke. Comparaison entre observation et prévision de la capacité portante et des tassements de pieux isolés battus dans une argile raide et fissurée à partir des essais pressiométriques Sols Soils $n^{\circ} 3$. 1980.

[4] De Beer. Lousbert et all Analysis of the results of loading tests performed on displacement piles of different types and sizes penetrating at a relatively small depth into a small dense sand laver. Proceeding of the conference. I. C.E. London 1979.

[5] Document Technique Unifié. $n^{\circ}$ 13.2. Travaux de fondations profondes pour le bâtiment. CSTB. Juin 1978.

[6] M. Bustamante, D. Gouvenot. Incidence des conditions d'exécution et du délai de repos sur le comportement et la portance des fondations. Revue Française de Géotechnique $n^{\circ} 6.1979$.

[7] J.-F. Jezequel, J.-N. Guegan, P. Liberge. Appareillage amovible pour la mesure des relations contraintes - déformations dans les pieux. Bulletin de liaison des LPC $\mathrm{n}^{\circ}$ 57. Janvier-Février 1972.

[8] J.-F. Jezequel, M. Bustamante. Mesure des élongations dans les pieux et tirants à l'aide d'extensomètres amovibles. Revue Travaux. Décembre 1972.

[9] Projet de mode Opératoire de l'Essai Statique de Fondations Profondes LCPC. Mai 1979.

[10] H. Cambefort, R. Chadeisson. Critère pour l'évaluation de la force portante d'un pieu. Proc. Sth. Int. Conf. Sol Méch. Found. Eng. 1961.

[11] M. Bustamante. Capacité d'ancrage et comportement des tirants injectés, scellés dans une argile plastique. Thèse de doctorat. ENPC 16.12.80.

[12] L. Ménard. Calcul de la force portante des fondations sur la base des résultats des essais pressiométriques. Sols Soils $\mathrm{n}^{\circ} 5$. Juin 1963.

[13] F. Baguelin, J.-F. Jezequel, D.-H. Shields. The pressuremeter and foundations engineering. Trans. Tech. Publications 1978.
[14] Fond 72. Fondations courantes d'ouvrage d'art LCP C.-SETRA. Février 1972.

[15] F. Baguelin, B. Gaudin. Essai de chargement d'un puits de fondation au viaduc du Pescaire. Bulletin Spécial des LPC «Autoroute de Menton ». Déc. 1971.

[16] O. Combarieu. Essais de chargement des pieux forés dans un limon argileux. Bulletin de liaison des $P$. et Ch. $n^{\circ} 80$. Nov.-Déc. 1975.

[17] O. Combarieu. Essais de chargement de pieux de grande longueur, battus dans de la grave et de l'argile raide. Bulletin de liaison des $P$ et $C h . n^{\circ} 82$ Mars-Avril 1976.

[18] M. Bustamante, M. Dervaux, Y. Hulo. Essais de chargement statique de 3 pieux lancés battus. Bulletin de liaison des P. et Ch. $\mathrm{n}^{\circ}$ 84. Juil.-Août 1976

[19] M. Bustamante, D. Gouvenot. Essais de pieux de haute capacité scellés par injection sous haute pression. Congrès de Vienne 1976.

[20] M. Bustamante, M. Panet. Essais statique d'arrachement d'un pieu M.-V. Trindel. Bulletin de liaison des P. et Ch. $n^{\circ} 79$. Sept.-Oct. 1975.

[21] M. Bustamante, L. Gianeselli, E. Waschkowski. Contrôle de la portance d'un pieu foré dans le calcaire de Beauce. Bulletin de liaison des P. et Ch. $n^{\circ} 106$. 1980.

[22] M. Bustamante, L. Gianeselli. Portance d'un pieu foré-tubé dans le calcaire de Saint-Ouen. Bulletin de liaison des $P$. et Ch. $n^{\circ} 107.1980$

[23] M. Bustamante, O. Combarieu, L. Gianeselli. Portance des pieux dans la craie altérée. Annales de I'ITBTP $n^{\circ}$ 174. Nov. 1980.

[24] J.-P. Bru. Problèmes d'exécution des pieux forés. Bulletin de liaison des P. et Ch. $n^{\circ} 77$. Mai-Juin 1975.

[25] «Les pieux forés". Recueil des règles de l'art. Direction des routes et de la circulation routière. Publication LCPC-SETRA. Déc. 1978.

[26] M. Bustamante, L. Gianeselli. Prévision de la capacité portante des pieux isolés sous charge verticale. Bulletin de liaison des P. et Ch. $n^{\circ} 113$.

[27] M. Gambin. Calcul du tassement d'une fondation profonde en fonction des résultats pressiométriques. Sols Soils $\mathrm{n}^{\circ} 7$. Déc. 1963.

[28] M. Cassan. Le tassement des pieux. Synthèses de recherches récentes et essais comparatifs. Sols Soils n $18 / 19 / 20.1966$. 